\title{
Towards a Modular Elbow Exoskeleton: Concepts for Design and System Control
}

\author{
Samet Ersoysal, Niclas Hoffmann, Lennart Ralfs \\ and Robert Weidner
}

\begin{abstract}
In industrial workplaces, strenuous, repetitive, and long-term tasks at head level or above as well as carrying heavy loads may lead to musculoskeletal disorders of different task dependent body parts. With an increasing trend towards wearable support systems, there is already a large quantity of exoskeletons that may support the user during movements, or stabilize postures, in order to reduce strain on various parts of the body. However, most commercially available exoskeletons mainly focus on the back and shoulder support. Only a few of them address the elbow joint, despite it being prone to injury. Therefore, this paper discusses different possible design and control concepts of modular elbow exoskeletons. The modular architecture potentially enables coupling to existing commercial- and research-associated systems, through appropriate interfaces. Different morphological structures and control mechanisms are assessed in respect to their ability to extend common exoskeletons for back and shoulder support.
\end{abstract}

S. Ersoysal $(\bowtie) \cdot$ N. Hoffmann $\cdot$ L. Ralfs $\cdot$ R. Weidner

Chair of Production Technology, University of Innsbruck, Institute of Mechatronic, Innsbruck, Austria

e-mail: Samet.Ersoysal@uibk.ac.at

N. Hoffmann

e-mail: Niclas.Hoffmann@uibk.ac.at; Niclas.Hoffmann@hsu-hh.de

L. Ralfs

e-mail: Lennart.Ralfs@uibk.ac.at

R. Weidner

e-mail: Robert.Weidner@uibk.ac.at; Robert.Weidner@hsu-hh.de

N. Hoffmann $\cdot$ R. Weidner

Laboratory of Manufacturing Technology, Helmut-Schmidt-University/University of the

Federal Armed Forces Hamburg, Hamburg, Germany

T. Schüppstuhl et al. (eds.), Annals of Scientific Society for Assembly,

Handling and Industrial Robotics 2021,

https://doi.org/10.1007/978-3-030-74032-0_12 
Based on these considerations, a first functional passive prototype is presented, which supports the flexion of the elbow joint and can be coupled to an existing exoskeleton. In future work, the prototype may be used for further elaboration and practical investigations in laboratory settings to evaluate its technical functionality and biomechanical effects on the user.

\section{Keywords}

Exoskeletons $\cdot$ Modular design $\cdot$ Support systems $\cdot$ Elbow $\cdot$ Wearable technologies Human-machine interaction

\section{Introduction}

Despite Industry 4.0 and increasing usage of semi or fully automated systems in production lines or logistics, employees still face non-ergonomic work conditions [1, 2]. Tasks in strenuous positions, with repetitive movements, and/or heavy-load-handlings, are associated with work-related musculoskeletal disorders [3]. A feasible solution to support manual tasks may be provided using exoskeletons that physically support different bodily regions of the user [4]. According to a systematic review of exoskeletons with near market maturity [5], there are 25 systems for the upper extremities, 10 systems for the lower extremities, and 26 systems for the back. It is shown, that only $34 \%$ of these systems address the elbow joint, because many exoskeletons for the upper extremities only support the neck-and-shoulder-region, with the path of force ending at the upper arm (e.g., exoskeletons like Lucy [6], Airframe [7], and Paexo Shoulder [8]). Any extension to the forearm/wrist implies taking multiple joints with their specific motion patterns and degrees of freedom into consideration, resulting in a more complex kinematic chain, that must be technically reproduced [9]. To bypass this issue, other systems feature a distal path of force, like third-arm-solutions (e.g., [10]), or direct draglines from the shoulder mount to the fingers/wrist (e.g., [11, 12]), which both results in systems being limited in their application, and often tailored to one specific use case. Furthermore, exoskeletons for back support, such as Laevo [13], CrayX [14], or Rakunie [15], often only support work in a bent-over position, or just lifting but not carrying of heavy loads.

Consequently, there is a demand for modularly extensions for existing exoskeletons to support the elbow joint in an additional way, since it is particularly burdened during manual repetitive tasks, as well as the carrying, and holding of heavy goods (e.g., loads and tools). In case of an overload, it is prone to an array of injuries, such as arthritis, biceps bracii tendon rupture, or lateral epicondylitis [16]. Here, the support particularly required in vertical direction, against gravitational forces in respect to support movements elevation and stabilize during static tasks is required. This paper shows and assesses 
different variants of proximal elbow support systems. For illustration, the preferred solution is presented as a first functional prototype.

\section{Determination of Main Requirements}

In general, support situations are influenced and determined by several dimensions, some of them are illustrated in Fig. 1.

For the design of a support system, the interaction of user, task, technology, and environment must be considered. Technical design principles for exoskeletons do exist (e.g., [17] and [18]), that address, e.g., safety, ergonomics and comfort, technical affinity, and usability. Additionally, the modular elbow support system follows the idea of Human-Hybrid-Robot [19], in order to reduce the harmful overload on certain body parts, by adapting support systems to different support demands. Thus, an analysis of existing support systems, in terms of their morphology and technical function, is essential to determine a suitable system structure and to ensure the later ability for connection. Furthermore, it is necessary to consider beforehand, the characteristics of the intended tasks and the end-user.

\subsection{Analysis of Connectable Exoskeletons}

Since various systems on the market, or in research-fields exist [5], only the proximal systems Lucy [6], Airframe [7], and Paexo Shoulder [8], for the upper extremities as well as Laevo [13], CrayX [14], and Rakunie [15] for the back are further analyzed here, in terms of their morphology (see Table 1). These systems feature physical interfaces such as

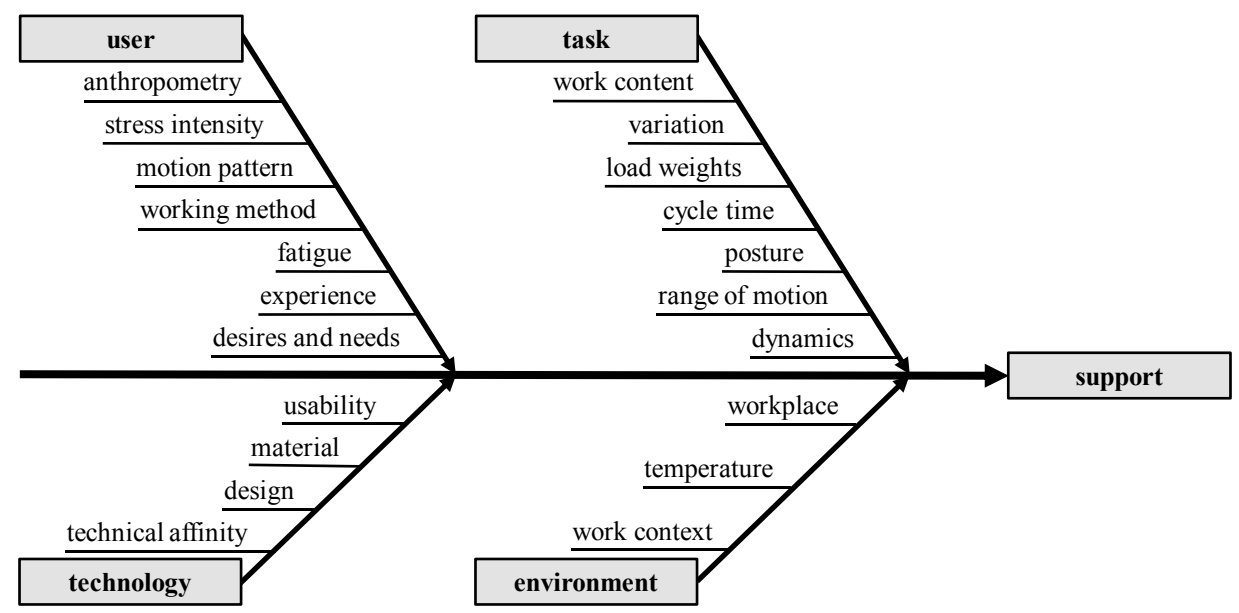

Fig. 1 Fishbone diagram of major factors affecting support situations 
Table 1 Overview of the mentioned exoskeletons with main characteristics

\begin{tabular}{|c|c|c|c|c|c|c|}
\hline Exoskeleton & Lucy & Airframe & $\begin{array}{l}\text { Paexo } \\
\text { shoulder }\end{array}$ & Laevo & CrayX & Rakunie \\
\hline $\begin{array}{l}\text { Supported } \\
\text { bodily region }\end{array}$ & \multicolumn{3}{|c|}{ Upper extremities } & \multicolumn{3}{|l|}{ Lower back } \\
\hline $\begin{array}{l}\text { Type of } \\
\text { actuation }\end{array}$ & $\begin{array}{l}\text { Active, } \\
\text { pneumatic }\end{array}$ & $\begin{array}{l}\text { Passive, } \\
\text { springs }\end{array}$ & $\begin{array}{l}\text { Passive, } \\
\text { springs }\end{array}$ & $\begin{array}{l}\text { Passive, } \\
\text { springs }\end{array}$ & $\begin{array}{l}\text { Active, } \\
\text { electric }\end{array}$ & $\begin{array}{l}\text { Passive, } \\
\text { elastic } \\
\text { bands }\end{array}$ \\
\hline $\begin{array}{l}\text { Physical } \\
\text { interface }\end{array}$ & Circular & Half-open & Circular & Half-open & Circular & Circular \\
\hline $\begin{array}{l}\text { System } \\
\text { weight }\end{array}$ & $5 \mathrm{~kg}$ & $2.5 \mathrm{~kg}$ & $1.9 \mathrm{~kg}$ & $2.8 \mathrm{~kg}$ & $8 \mathrm{~kg}$ & $0.25 \mathrm{~kg}$ \\
\hline Structure & Rigid & Rigid & Rigid & Rigid & Rigid & Soft \\
\hline
\end{tabular}

shoulder straps, and most commonly a belt around the pelvis, for both carrying the systems' weight, and applying and transferring supporting forces to stronger, stabilizing body parts. These forces are often transferred via a proximal back structure, that can be composed of either rigid or soft elements. In some cases, this structure is shifted to the sides (see Paexo Shoulder [8]) or to the abdomen (see Laevo [13]).

\subsection{Analysis of Intended Tasks}

The intended tasks that should be supported by the elbow system are related to carrying heavy loads or working with heavy tools, in particular, since they are factors contributing to work-related musculoskeletal disorders [2]. It must be considered, that the work environment may be in confined, moist, or rough conditions as well as require the user to wear mandatory protective equipment, such as gloves, or jackets. When looking at working techniques for carrying loads, it becomes obvious, that humans often use the lower part of their inner forearm, either as an additional shelf space to relieve stress on the wrist, or as a clamp, to decrease the grasping force of the fingers. According to the working position for this task, it can be said, that the forearm is normally bent at a $90^{\circ}$ angle or an almost fully extended position. When holding heavy tools with both hands, it is also possible, that one elbow may be hyperextended (ca. $135^{\circ}$ ) and the other in flexion (less than $90^{\circ}$ ), meaning that the joint angle varies between both arms. Also, a lot of tasks are performed with both hands in use, which requires manual control of the exoskeleton.

\subsection{Analysis of Human Anthropometry and Kinematics}

The human anthropometry is important for the fit of the system. Different studies for body proportions do exist. For instance, Winter et al. [20] estimate the length of the upper arm 
and the forearm, in relation to the users' height. Alternatively, Dempster et al. [21] published different data for an array of different body parts, for length, weight, and the center of mass, which may give an appropriate foundation for further development concerning dimensions of the system. In order to maintain the users' natural movement while wearing the system, human biomechanics should also be considered. The elbow joint complex consists of the elbow joint and the radioulnar joints and has two degrees of freedom: flexion/extension and supination/pronation [18]. The flexibility of healthy individuals is $0^{\circ}$ to $140^{\circ}$ (flexion/extension), $85^{\circ}$ (supination) and $75^{\circ}$ (pronation). A hyperextension of $5^{\circ}$ to $10^{\circ}$ in flexion/extension is not seldom [16]. According to comfort, the design, position, and clamp of the interfaces are also important. Principally, interfaces should be designed more broadly, to distribute contact pressures better to the human skin. Avoidable areas of contact are principally the joints themselves (elbow, wrist) and their immediate vicinity (especially medial and lateral epicondyle) [17, Fig. 5.21]. The contact pressure also needs to be limited, because it may significantly constrict the blood supply to the arm [22]. In order to avoid interaction forces, any misalignment of the joints between the exoskeleton and human limb should also be neglected [17].

\section{Design and System Control Concepts}

Different approaches for supporting the elbow joint are possible. The systems may morphologically vary, mainly in terms of path of force, rigidity of the structural elements, and type of actuation [6], which naturally determines different technical properties and effects on the wearer [4]. In the following, different basic morphological concepts are derived or inspired from already existing approaches, as well as assessed in terms of their suitability for the introduced application field.

\subsection{Design Approaches}

In general, it is advisable for exoskeleton development to begin with the actuation of only one degree of freedom, in order to maintain users' flexibility, and to reduce the construction complexity [9]. Thus, elbow support systems often only address the more exhausting flexion. Figure 2 clusters different ways of design and control for proximal exoskeletons supporting the elbows joints' flexion. Each group can naturally have individual configurations by experts, but the basic principle stays the same:

- Approach 1: According to the design, approach one uses tensile forces with a circular interface on the forearm and a rope guide on the upper arm, e.g., [23, 24]. Here, the actuator is often placed on the upper back and the force is transmitted by a Bowden cable. Alternatively, the actuator can be directly attached to the upper arm. A biomimetic design with artificial muscles is also possible. 


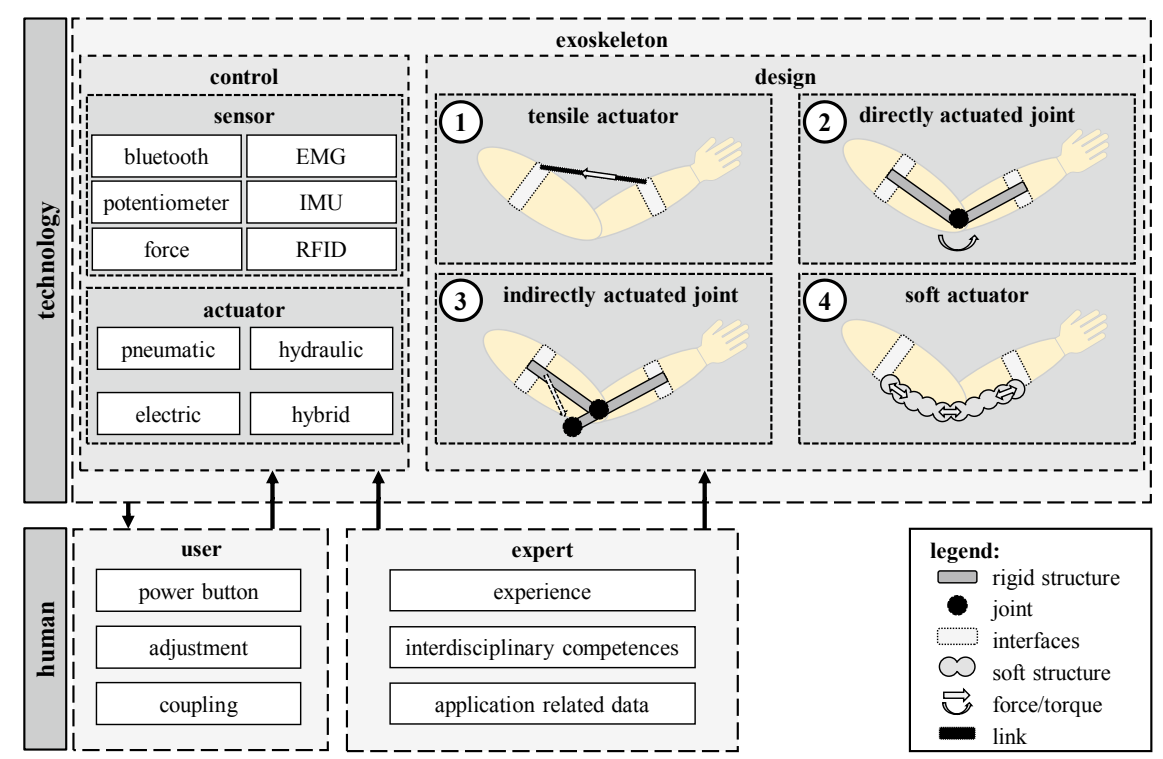

Fig. 2 Considerable design and control strategies for elbow support systems

- Approach 2: A possible way is using a directly actuated joint, consisting of two rigid bars that are respectively fixed to the upper arm and forearm, e.g., [25]. Here, the actuator can generate a direct torque, to support the flexion/extension of the elbow.

- Approach 3: An indirectly actuated joint could be realized by transferring the actuation principle from, e.g., Lucy [6] to the elbow joint, whereas the actuator may be attached to either the upper arm, or the forearm. In this case, the torque, or support, is generated according to the lever arm principle.

- Approach 4: A soft alternative are pneumatically inflatable air cushions, e.g., [26, 27]. A design with soft cylindrical actuators arranged in an array, generating a torque when inflated. A different approach of a soft support system are chamber elements for stabilizing body postures, e.g., [28].

\subsection{System Control Strategies}

The control mechanisms determine the steering of the systems' support, by either the user, the system itself, or an expert. It depends on whether the system is actuated in a passive or active way. Passive exoskeletons use actuators like springs with by experts pre-determined relations, between the supporting force and the arm angle, or the position of the actuator. Here, the user can sometimes decouple the actuation from the system for subsidiary tasks or pauses, as well as make some adaptions to the curve of force by, e.g., changing the preload of the springs (see, e.g., Paexo Shoulder [8]), or exchanging the 
actuator (see, e.g., Airframe [7]). Alternatively, active exoskeletons determine the arm position with sensors, like inertial measurement units (IMU) [29] or linear potentiometers [6]. The support demand, dependent on behavior or situation, can be identified by force sensors in gloves [25], by measurements of the muscular activity for grabbing via EMG-sensors [30], or by the determination of the load (e.g., product, ware, tool) via RFID or Bluetooth [31,32]. Based on this information, experts can pre-determine the necessary support of the system. Also, the user can, adjust the level of support directly with a control element, or by actuating a power button [6].

\subsection{Assessment in View to Modularity and Intended Tasks}

In reference to the analyzed morphology of the exoskeletons that should be modularly extended and the described application field (see Chap. 2), a qualitative evaluation and comparison of the four described design approaches is made (see Table 2).

It is shown that a support mechanism working with tensile forces (mechanism 1 in Fig. 2) seems to be beneficial for a modular design. In comparison to mechanism 2 and 3 , the first mechanism can easily be realized in a soft execution. The soft structure enables wearing the elbow system underneath other systems and tensile forces can easily be proximally extended, to place the actuator to regions, where it does not disturb existing

Table 2 Qualitative evaluation of the presented design concepts according to various criteria

\begin{tabular}{|c|c|c|c|c|}
\hline properties & approach 1 & approach 2 & approach 3 & approach 4 \\
\hline \multicolumn{5}{|l|}{ use cases } \\
\hline support static posture & 0 & 0 & O & (1) \\
\hline support dynamic movements & 0 & (1) & 0 & $\bigcirc$ \\
\hline \multicolumn{5}{|l|}{ support characteristic } \\
\hline level of support & (1) & 0 & 0 & O \\
\hline force adjustment & (1) & 0 & 0 & O \\
\hline \multicolumn{5}{|l|}{ ergonomic aspects } \\
\hline weight at distal end & O & O & ( & ( \\
\hline wearability/comfort & 0 & (1) & ( & (1) \\
\hline \multicolumn{5}{|l|}{ construction } \\
\hline modular coupling & - & () & (1) & (1) \\
\hline individual adjustment & 0 & 0 & 0 & O \\
\hline \multicolumn{5}{|l|}{ averaged rating } \\
\hline$\bigcirc 1$ (low) & () 2 & (1) 3 & O 4 & 5 (high) \\
\hline
\end{tabular}


structures, and keeps the additional mass on the arm as low as possible [33]. This is technically not possible for the principles of directly (2) and indirectly (3) actuated joints, since rigid structures are required for torque transmission. Soft actuators (4) could have potential as well, but often imply as pneumatically driven active systems both complex control schemes and external energy supplies, e.g., [26]. Here, the modular elbow support system should better be passive in order to keep the modular extension as simple as possible, to reduce the total weight and the amount of needed system elements, and to ease the general connectivity. Active systems simply influence existing systems greater, by changing total system characteristics, particularly from passive systems when adding sensors, energy storages, and control units.

\section{Realization of Functional Prototype}

The functional prototype (see Fig. 3) is inspired by, e.g., [11, 23, 24] for the structure, elements, and path of force, but changes some vital characteristics for a better modular fit, since these systems are principally designed for being autonomous. In this context, it is essential to ensure that the elements of the developed system do not interfere with other systems. Thus, the path of force on the upper arm is longer proximal than, e.g., [11] to use the interface of the analyzed systems for the upper extremities without any problems. However, this reduces the effectiveness of the tensile forces to induce a supporting torque on the elbow joint [34]. The actuator also moves to the pelvis belt, by extending the tensile lines, due to most systems already having one pelvis belt, interferences with the back structure are avoided, and the pelvis region is appropriate for taking tensile forces. Furthermore, the dual use of possibly existing pelvis belts leads to a reduction of the number of elements and the systems' weight.

The general path of the tensile forces is orientated by the line of non-extension [35] for better effectiveness. A steel rope is used that can be easily adjusted to different body dimensions, by wire rope clips, both near the elbow and on the back. The path proceeds

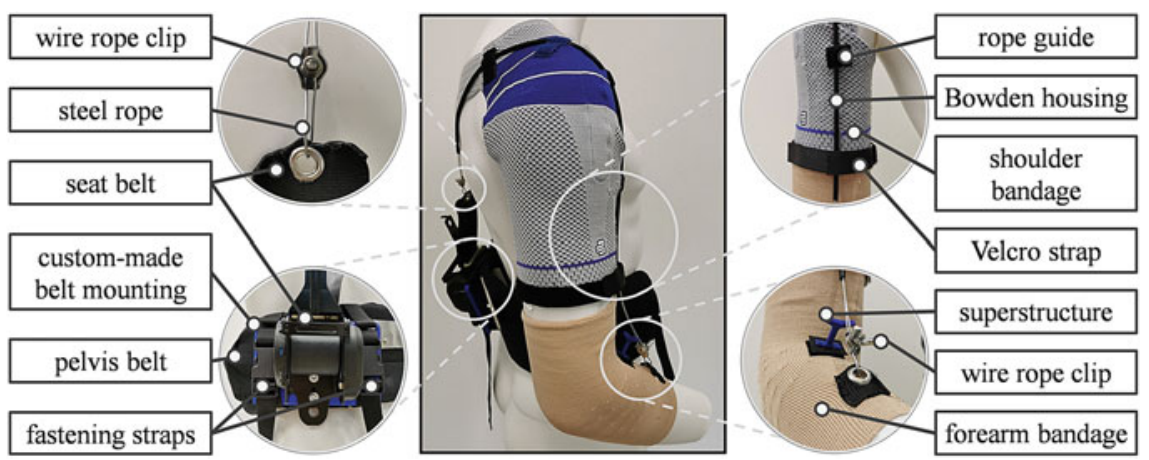

Fig. 3 Overview and central elements of the modular soft elbow support system 
from a loop on the forearm bandage over the shoulder bandage, to the pelvis adapter. An installed Bowden housing on the shoulder bandage, as in [29] reduces rubbing and keeps the rope in position. The medical shoulder bandage is extended by a Velcro strip on the upper arm to avoid a protruding of the Bowden cable. The point of force origin on the forearm is also more orientated towards the elbow joint, to keep the lower forearm free and to maintain the typical working techniques. The prototype uses a medical arm bandage, since the use of neoprene interfaces help to reduce contact pressures [36]. Due to the human soft tissue and the soft structure of the connecting element between the bandage around the upper and the forearm, a movability in supination/pronation is preserved. A new-designed superstructure is connected to the forearm bandage, and maintains an induced support torque, by the tensile forces even when the arm is almost fully extended [34].

The actuator is fixed to the pelvis belt via a custom-made mounting. Here, a lock mechanism is preferred, which can lock in different length, in order to support the user in various elbow joint angles, or arm positions. The mechanism is firstly represented by a seat belt of a car, as seen in [34]. Thus, the mechanism locks if the belt is quickly dragged out and disengaged with a little move in the other direction, which means that the user can trigger it on demand. On the back, the belt passes into the steel rope with a hook and eye principle. The custom-made mounting can be easily mounted and tightened to pelvis belts of various widths, by using fastening straps and side-release buckles.

\section{Conclusion and Outlook}

Although the elbow joint is burdened in many industrial tasks, many exoskeletons for back and shoulder support do not cover it. The presented modular elbow support system may close this gap, by being able to be coupled to such systems and support the flexion of the elbow joint, with induced tensile forces. In the future, a comprehensive laboratory study should be conducted, in order to estimate its technical functionality and biomechanical effects on the wearer. Furthermore, the systems' connectivity to various existing exoskeletons should be checked. Effort could also be made to improve the lock mechanism in its sensitivity, and to merge the elements of the modular system, e.g., both arm bandages.

However, the lock mechanism is limited to static tasks, although many tasks are dynamically performed with different arm positions. Here, an active actuated modular extension of active support systems like, e.g., Lucy [6] could be beneficial and extend possible application cases. Consequently, it is recommended to persist with this concept as well. 


\section{References}

1. Bogue, R.: Exoskeletons - a review of industrial applications. Ind. Robot. Int. J. 45(5), 585590 (2018)

2. Eurofound: Sixth European Working Conditions Survey - Overview report (2017 update). Publications Office of the European Union, Luxembourg (2017)

3. Sluiter, J.K., Rest, K.M., Frings-Dresen, M.H.: Criteria document for evaluating the work-relatedness of upper-extremity musculoskeletal disorders. Scand. J. Work Environ. Health 27, 1-102 (2001)

4. Fox, S., Aranko, O.: Exoskeletons: Comprehensive, comparative and critical manufacturing performance. J. Manuf. Technol. Manag. (2019)

5. Weidner, R., Linnenberg, C., Hoffmann, N., Prokop, G., Edwards, V.: Exoskelette für den industriellen Kontext: Systematisches Review \& Klassifikation. Digitaler Wandel, digitale Arbeit, digitaler Mensch?, 66. Kongress d. Gesellschaft für Arbeitswissenschaften (2020)

6. Otten, B., Weidner, R., Argubi-Wollesen, A.: Evaluation of a novel active exoskeleton for tasks at or above head level. IEEE Robot. Autom. Lett. 3(3), 2408-2415 (2018)

7. Spada, S., Ghibaudo, L., Gilotta, S., Gastaldi, L., Cavatorta, M.P.: Investigation into the applicability of a passive upper-limb exoskeleton in automotive industry. Procedia Manuf. 11, 1255-1262 (2017)

8. Maurice, P., Ivaldi, S., Babic, J., Camernik, J., Gorjan, D., Schirrmeister, B., Bornmann, J., Tagliapietra, L., Latella, C., Pucci, D., Fritzsche, L., Ivaldi, S., Babic, J.: Objective and subjective effects of a passive exoskeleton on overhead work. IEEE Trans. Neural Syst. Rehabil. Eng. 28(1), 152-164 (2020)

9. Asbeck, A.T., Dyer, R.J., Larusson, A.F., Walsh, C.J.: Biologically-inspired soft exosuit. IEEE Int. Conf. Rehabil. Robot, 1-8 (2013)

10. Lockeed Martin: Fortis. Homepage. https://www.lockheedmartin.com/en-us/products/ exoskeleton-technologies/industrial.html. Last accessed 30 Aug 2020

11. Li, N., Yang, T., Yu, P., Chang, J., Zhao, L., Zhao, X., Elhajj, I.H., Xi, N., Liu, L.: Bio-inspired upper limb soft exoskeleton to reduce stroke-induced complications. Bioinspiration \& Biomimetics 13(6), (2018)

12. Strongarm Tech: V22 ErgoSkeleton. Homepage. Last accessed 25 June 2020

13. Koopman, A.S., Kingma, I., Faber, G.S., de Looze, M.P., van Dieen, J.H.: Effects of a passive exoskeleton on the mechanical loading of the low back in static holding tasks. J. Biomech. 83, 97-103 (2019)

14. German Bionic (2020). Cray X. Homepage. Last accessed 09 Sept 2020

15. N-ippin. Rakunie. Homepage. https://www.morita119.com/en/products/supportwear/rakunie/ 001.html (2020). Last accessed 09 Sept 2020

16. Vavken, P., Rosso, C.: Der schmerzende Ellbogen in der Praxis. Swiss Medical Forum Schweizerisches Medizin-Forum 17(44), 953-959 (2017)

17. Pons, J.L.: Wearable Robots: Biomechatronic Exoskeletons (2008)

18. Gopura, R.A.R.C., Kiguchi, K.: Mechanical designs of active upper-limb exoskeleton robots. In: IEEE 11th International Conference on Rehabilitation Robotics, pp. 178-187 (2009)

19. Weidner, R., Kong, N., Wulfsberg, J.P.: Human hybrid robot: a new concept for supporting manual assembly tasks. Prod Eng. 7(6), 675-684 (2013)

20. Winter, D.: Biomechanics and Motor Control of Human Movement, Fourth Edition (2009)

21. Dempster, W.T., Gaughran, G.R.L.: Properties of body segments based on size and weight. Am. J. Anat. 120(1), 33-54 (1967) 
22. Holloway, G.A., Daly, C.H., Kennedy, D., Chimoskey, J.: Effects of external pressure loading on human skin blood flow measured by 133Xe clearance. J. Appl. Physiol. 40(4), 597-600 (1976)

23. Dinh, B.K., Xiloyannis, M., Antuvan, C.W., Cappello, L., Masia, L.: Hierarchical cascade controller for assistance modulation in a soft wearable arm exoskeleton. IEEE Robot. Autom. Lett. 2(3), 1786-1793 (2017)

24. Chiaradia, D., Xiloyannis, M., Antuvan, C.W., Frisoli, A., Masia, L.: Design and embedded control of a soft elbow exosuit. In: IEEE International Conference on Soft Robotics (Robosoft), pp. 565-571 (2018)

25. Otten, B., Stelzer, P., Weidner, R., Argubi-Wollesen, A., Wulfsberg, J.P.: A novel concept for wearable, modular \& soft support systems used in industrial environments. In: Proceedings of the 49th Hawaii International Conference on System Sciences, pp. 542-550 (2016)

26. Otherlab. Homepage. https://www.otherlab.com (2015). Last accessed 22 Sept 2020

27. Thalman, C.M., Lam, Q.P., Ngyuen, P.H., Srider, S., Polygerinos, P.: A novel soft elbow exosuit to supplement bicep lifting capacity. In: IEEE/RSJ Iternational Conference on Intelligent Robots and Systems (IROS), pp. 6965-6971 (2018)

28. Weidner, R., Meyer, T., Argubi-Wollesen, A., Wulfsberg, J.P.: Towards a modular and wearable support system for industrial production. Appl. Mech. Mater. 840, 123-131 (2016)

29. Lessard, S., Pansodtee, P., Robbins, A., Trombadore, J.M., Kurniawan, S., Teodorescu, M.: A soft exosuit for flexible upper-extremity rehabilitation. IEEE Trans. Neural Syst. Rehabil. Eng. 26(8), 1604-1617 (2018)

30. Koopman, A.S., Toxiri, S., Power, V., Kingma, I., van Dieën, J.H., Ortiz, J., de Looze, M.P.: The effect of control strategies for an active back-support exoskeleton on spine loading and kinematics during lifting. J. Biomech. 91, 14-22 (2019)

31. Weidner, R., Matthiesen, S., Bruchmüller, T., Mangold, S., Wulfsberg, J.P.: Systematische Entwicklung von Einheiten aus Power-Tools und anziehbaren Unterstützungssystemen Ansatz einer integrativen Entwicklung. In: Weidner, R. (ed.) (Hg.): Band zur 2. Transdisziplinäre Konferenz „Technische Unterstützungssysteme, die die Menschen wirklich wollen“, pp. 527-534 (2016)

32. Bances, E., Schneider, U., Siegert, J.T.B.: Exoskeletons towards industrie 4.0: benefits and challenges of the IoT communication architecture. Procedia Manuf. 42, 49-56 (2020)

33. Browning, R.C., Modica, J.R., Kram, R., Goswami, A.: The effects of adding mass to the legs on the energetics and biomechanics of walking. Med. Sci. Sports Exerc. 39(3), 515-525 (2007)

34. Hoffmann, N., Weidner, R., Schubert, T., Wulfsberg, J.P.: Towards a soft shoulder support system. In: Schüppstuhl, T., Tracht, K., Roßmann, J. (eds.) Tagungsband des 4. Kongresses Montage Handhabung Industrieroboter. Springer, Heidelberg (2019)

35. Iberall, A.S.: The use of lines of nonextension to improve mobility in full-pressure suits: AMRL-TR. Aerosp. Med. Res. Lab. (6570th), 1-35 (1964)

36. Xiloyannis, M., Chiaradia, D., Frisoli, A., Masia, L.: Characterisation of pressure distribution at the interface of a soft exosuit: towards a more comfortable wear. In: Carozzo, M., Micera, S., Pons, J. (eds.) Challenges and Trends. WeRob 2018. Biosystems \& Biorobotics 22, Springer (2019) 
Open Access This chapter is licensed under the terms of the Creative Commons Attribution 4.0 International License (http://creativecommons.org/licenses/by/4.0/), which permits use, sharing, adaptation, distribution and reproduction in any medium or format, as long as you give appropriate credit to the original author(s) and the source, provide a link to the Creative Commons license and indicate if changes were made.

The images or other third party material in this chapter are included in the chapter's Creative Commons license, unless indicated otherwise in a credit line to the material. If material is not included in the chapter's Creative Commons license and your intended use is not permitted by statutory regulation or exceeds the permitted use, you will need to obtain permission directly from the copyright holder.

(c) (1) 